\title{
Une lettre inédite de Sade
}

\author{
Daniel Celce-Murcia
}

Depuis 1833 environ, des fragments de 1'oeuvre de Sade (manuscrits, lettres, documents en tous genres) ne cessent de s'accumuler. On peut en constater l'étendue dans la remarquable série des oeuvres de Sade éditée par Gilbert Lely ${ }^{1}$, mais de continuelles découvertes apportent--ne fût-ce que par leur valeur. anecdotique--un élément précieux pour faire revivre le dix-huitième siècle. La lettre que nous reproduisons ici en est un exemple. 2

Elle se rapporte à un des nombreux épisodes judiciaires de la vie du Marquis de Sade. Nous avons pu la dater du mercredi 12 juillet 1797 (24 messidor an $\mathrm{V})^{3}$, sept ans après sa libération de Charenton (2 avril 1790) et 1 'année même de la parution de La Nouvelle Justine. Au mois de mai 1797, Sade quitte Paris pour la Provence. Il a vendu en octobre 1796 son domaine de La Coste mais il est en dispute avec le nouveau propriétaire, J.S. Rovère. Mme de Sade, qui voudrait récupérer sa dot et qui se méfiait des transactions de son mari, a pris des sûretés de conservation hypothécaire sur la vente de La Coste. Pour régler les formalités nécessaires, elle a dépêché en Provence son fils aîné que le marquis accuse aussitôt de calomnie: "c'est un vilain drôle que M. de S. fils. Ce qu'.on appelle un vilain b..." 
A Mazan, la municipalité vient visiter en corps le marquis, mais place chez lui un "garnisaire", qui lui "mangera" cinq ou six jours de plus qu'il ne faut, pour le contraindre à payer ses impôts. 4 Au milieu de ces démêlés, Sade, alors à Saumane, se met une nouvelle affaire sur le dos: c'est à cet incident que notre lettre se rapporte. En effet, le 18 juin (30 prairial) Sade accuse de forfaiture le citoyen Noël Perrin, receveur de 1 'enregistrement et du domaine national à Carpentras. "Vous n'avez pas osé faire passer dans les coffres de la nation la rente d'un homme qui n'a jamais émigré de sa vie; elle ne l'aurait pas reçue. Cette rente n'a donc été versée que dans votre poche", 5 lui écrit-il. Il parle même de le dénoncer au Directoire. Devant le silence de Perrin, Sade se sent déjà vainqueur. Mais Perrin, qui n'est pas coupable, ne peut se laisser accuser impunément. Le 8 juilllet (20 messidor), il réplique aux accúsations de Sade en le citant devant le tribunal correcitonnel d'Avignon, (séance du samedi 15 juillet à dix heures), 6 afin que soient déclarés "faux et calomnieux" tous les faits contenus dans les deux lettres de Sade. 7

Sade sentant que I'affaire tourne mal, décide de se rétracter, avant que sa cause ne passe devant le tribunal. Le mercredi 12 juillet (24 messidor), Mâ̂tre Liotard, notaire à l'Isle-sur-Sorgue, doit passer à Saumane dresser 1'acte de désaveu (paragraphe 5 de la lettre: "Liotard de 1'Isle va arriver pour dresser 1'acte..."). Mais Sade avait peut-être déjà rédigé 1 'acte dans ses grandes lignes (paragraphe 1 de la lettre: "L'acte ci-joint vous dit ce que c'est..."). Toujours est-il, qu'en attendant Liotard, Sade écrit à Gaufridy, son avocat de longue date, pour le mettre au courant des derniers développements de ses affaires. L'affaire Perrin ne semble pas gêner Sade outre-mesure. E1le est même pratiquement close en ce qui le concerne. 8 I1 a "d'autres chats à fouetter"! I1 va repartir 
pour trois semaines et essayer de se procurer de 1 'argent par tous les moyens.9

Sade veut encore, d'après sa lettre, vendre les prés de St Pol (ou St Paul) dont il obtiendra, semble-t-il, une somme dérisoire. 10

Mais la préoccupation dominante de cette lettre du 12 juillet est une affaire qu'il a en tête probablement depuis quelque temps, affaire peu ordinaire et pour laquelle il a besoin de Charles Gaufridy qu'il réclame avec insistance à son père. En effet la foire de Beaucaire s'ouvre le 22 juillet et Sade veut y organiser une loterie dont il attend un grand profit. Il veut envoyer Charles en avance pour y vendre les billets. Mais le 23 juillet quand Sade rejoindra Charles en compagnie de François Gaufridy, son frère, il sera si déçu que Charles n'ait vendu aucun billet qu'il le renverra à son père, chez qui il a laissé Mme Quesnet.

E1le lui manque beaucoup, et Sade "a hâte de revoir cette sincère amie dont 1 'existence lui est plus chère que la sienne."12

Nous avons fait l'impossible mon cher avocat pour vous épargner le voyage d'Avignon; messieurs Mestre? et Connifri? se sont conduits d'après vos vues et $n^{\prime}$ ont absolument rien pu obtenir. Perrin, conseillé sans doute, est d'une opiniâtreté sans exemple, il ne veut entendre absolument à rien. Nous avons, d'après cela, suivi littéralement le document de M. Mézard à qui vous avez bien voulu m'adresser. Voilà une lettre de lui que je n'ai pas lue et par laquelle sans doute il vous trace la conduite qu'il nous a conseillée et que nous suivons. L'acte ci-joint vous dit ce que c'est et m'évite la peine de vous le répéter. I1 est donc absolument essentiel que vous ayez la complaisance d'aller à Avignon signifier vous-même cet acte au tribunal ou à Perrin samedi matin; Je vous 
conjure de revenir dîner à Saumane dimanche, attendu que je pars lundi pour un voyage de trois semaines, et que je veux absolument vous voir et vous parler avant que de partir; il le faut même de toute nécessité. Je vous attends donc sans faute dimanche à dîner. Puis adieu jusqu'au 6 août époque où nous nous réunirons à Saumane pour vos comptes, ceux de Ripert et ceux de Lions.

Vous m'avez exactement coupé les bras et les jambes. Vous me faites exactement manquer mon affaire d'Arles en ne m'envoyant pas Charles tout de suite comme je vous en avais supplié. Tout sera manqué. L'homme en question aura peut-être été à Arles, nous voilà? [un mot illisible] reserré comme le diable par Lombard auquel la ferme est adjugée le 1 août si l'on n'a rien terminé avant... Oh! Juste ciel, quel contretemps pour moi que ce maudit retard. Ah! Enfin qu'il arrive donc sans faute au moins demain, je vous en conjure, afin d'être au poste samedi. S'il n'arrive que samedi, voilà encore deux jours de perdus car il ne fait rien dimanche. C'est à genoux, exactement à genoux que je vous supplie de ne plus mettre le moindre retard au départ de Charles. Je 1'attends sans faute à dîner demain vendredi.

Ces messieurs sont arrivés mécontents de Mazan. L'homme en question ne s'en tient pas à publier tout ce qu'on peut dire pour s'opposer, il ose encore répandre $I^{\prime}$ infâme calomnie que mes affaires sont très mauvaises à Paris, où il est de fait que je n'ai d'autres dettes que deux mille écus dont mille empruntés pour faire mon voyage et mille pour achever le payement de 1 'une de mes nouvelles terres achetées. Est-ce 1à devoir? Sont-ce là de mauvaises affaires? Oh! C'est un vilain drôle que M. de S. fils. Ce qu'on appelle un vilain b..., mais patience rira bien qui rira le dernier et je le remercie de me mettre au cas de n'avoir plus aucune espèce de ménagement pour lui.

Cependant ces messieurs ont, à force de parler, 
détruit quelques préjugés; on va voir.

Les prés de St Pol, affichés isolément par votre conseil, seront délivrés au plus offrant mardi 18; c'est pour cela que je pars lundi et que je veux vous voir dimanche.

Votre Voux nuit plus qu'il ne sert; c'est un fort vilain monsieur.

Courtois ne s'est point entendu avec lui, ou lui point avec Courtois et tout par ce moyen a été de travers car Courtois qui se conduit assez bien, a envoyé du monde voir les prés à Mazan et personne ici pour montrer ces prés-1à; il devait donc en prévenir Voux.

[deux mots--serrurier?] a été surveiller la récolte, le garnisaire est levé, le receveur de Mazan s'est bien conduit, on lui a donné un acompte et il a promis que si pareille chose arrivait encore, qu'il en donnerait avis sur le champ. Si Voux, le notaire, avait averti par un exprès, il n'y aurait rien eu; mais il lui a paru plaisant d'envoyer par la poste afin que le garnisaire me mangeât cinq ou six jours. C'est un mauvais homme que votre Voux.

Résultat. J'attends votre fils Charles demain vendredi à dîner. [un mot] dimanche; à votre retour d'Avignon ou j'aurai quelque chose de fort essentiel et de fort singulier à vous dire.

Mille et mille pardons mon cher avocat. Voilà un terrible été pour vous, je le sais, mais il y avait dix-neuf ans que je ne vous avais importuné et tout ceci fini j'en serai peut-être bien d'avantage sans que je revienne troubler votre repos et votre tranquillité. Cette remarque est de quelque considération. Liotard de l'Isle va arriver pour dresser 1'acte et je garde ce reste de papier ou pour de nouvelles réflexions s'il en survient ou pour vous embrasser.

A I'instant même je reçois une lettre de mon fils qui me dit qu'il part à la minute même pour 
Paris. Que dites-vous de toutes ces extravagances1 à?

N'oubliez donc pas de nous apporter cette [un mot] tant promise de Saumane, nous ne pouvons rien statuer sur cette terre sans cela.

N'oubliez pas d'apporter le chocolat, je vous prie.

Le commencement de ma lettre vous annonçait un acte qui devait y être joint et vous expliquer tout. Nous avions oublié en vous annonçant cela, la formalité nécessaire de faire signifier cet acte à Perrin. C'est à quoi va s'employer la journée de jeudi. Moyennant quoi, partez, je vous prie. Vendredi matin passez à I'Isle chez M Liotard, vous y trouverez I'acte qui viendra d'y être signifié et que vous emporterez avec vous à Avignon. Vous vous en servirez samedi et repasserez sans faute dimanche par Saumane en vous en retournant à Apt. N'oubliez pas cette circonstance, je vous en conjure, étant très essentiel que je vous voie: dimanche avant mon départ de lundi m.tin. Je dois être trois semaines absent et je ne puis absolument me passer de vous voir avant.

A travers de tout cela, n'oubliez pas que Charles vienne dîner vendredi à Saumane, cette clause est pour moi de la plus extrême importance.

$\mathrm{Je}$ vous accolle de ma reconnaissance et vous embrasse de tout mon coeur.

de Sade

Ce Mercredi au soir.

University of California

at Los Angeles 



\section{Notes}

${ }^{1}$ D.A.F. de Sade, Oeuvres Complètes, ed. Gilbert Lely (Paris: Jean-Jacques Pauvert, 1961).

${ }^{2}$ Cette lettre autographe fut découverte par mon ami le Docteur Stephen Werner, professeur à I'Université de Californie à Los Angeles, qui eut la gentillesse de $\mathrm{m}^{\prime}$ en communiquer une photocopie.

${ }^{3}$ Pour dater la lettre nous possédions trois indications de Sade: le départ du mardi 18, le retour du 6 août d'un voyage de trois semaines, Ia signature du mercredi précédent le mardi 18.

Le mercredi où la lettre avait été écrite devait donc être le mercredi 12, et le mois était alors juillet. Restait 1'année, année où Sade d'après le ton de la lettre, devait être en liberté. Voir pour cela Gilbert Lely, La Vie du Marquis de Sade avec examen de ses ouvrages, 2 vols. (Paris: Gallimard, 1957), II 497 et suivantes.

${ }^{4}$ Ibid, Voir aussi Paul Bourdin, Correspondance Inédite du Marquis de Sade, de ses proches et de ses familiers, publiée avec une introduction des annales et notes (Paris, 1929), p. 300.

${ }^{5}$ Lely, Vie du Marquis de Sade, II, 497.

6 Bourdin, Correspondance inédite, p. 405.

7 "Notamment 1'inculpation faite au requérant d'avoir détourné à son profit une partie des revenus du dit Sade, dont la recette a été par lui faite en sa qualité de receveur du domaine national et pour réparation, se voir condamner à la somme de quinze cents francs d'amende applicable à l'aumône de la commune d'Avignon, aux frais d'impression, publication et affiches du jugement...", avec 
défense de récidiver. (Bourdin, p. 406).

${ }^{8}$ Voici comment se termina 1'affaire Perrin: Lorsque Liotard arrive à Saumane le 12 juillet, il dresse 1'acte de désaveu dans lequel le Marquis "reconnaît le citoyen Perrin pour un homme probe et honnête, ayant toujours rempli les fonctions de son état avec distinction" (Bourdin, p. 407), et s'offre à payer les frais passés et à venir.

Le lendemain 13 juillet, Sade fait signifier son désaveu à Perrin par Lavagne, officier ministériel, dans l'étude du notaire Courtois. (Bourdin, p. 407).

Perrin par égard pour la famille de Sade et pour les personnes qui se sont employées à terminer cette affaire, accepte de se départir de la citation à condition que Sade paie les frais et dépens, qu'il consigne la somme nécessaire pour les frais d'impression et d'affiche de l'acte de rétractation et de sa réponse, qu'il verse une aumône à 1 'hôpital de Carpentras et qu'il éxécute tout cela dans les vingt-quatre heures. (Bourdin, p. 407408).

${ }^{9}$ Sade veut vendre son Mas de Cabanes, près d'Arles. "Toutefois le Marquis s'est déjà engagé avec son fermier Lombard qui doit lui verser 6.000 livres d'acompte. Cet argent sera bon à prendre sans que la chose mette obstacle à l'éxécution des autres projets, car 'vente casse vente' selon $M$. de Sade qui sait accomoder les principes du droit à son propre usage." (Bourdin, p. 400).

10 Bourdin, Correspondance inédite, p. 399.

${ }^{11}$ Lely, Vie du Marquis de Sade, p. 499.

12 Bourdin, Correspondance inédite, p. 400 . 


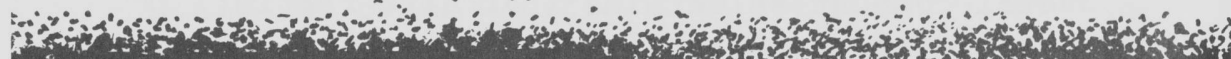
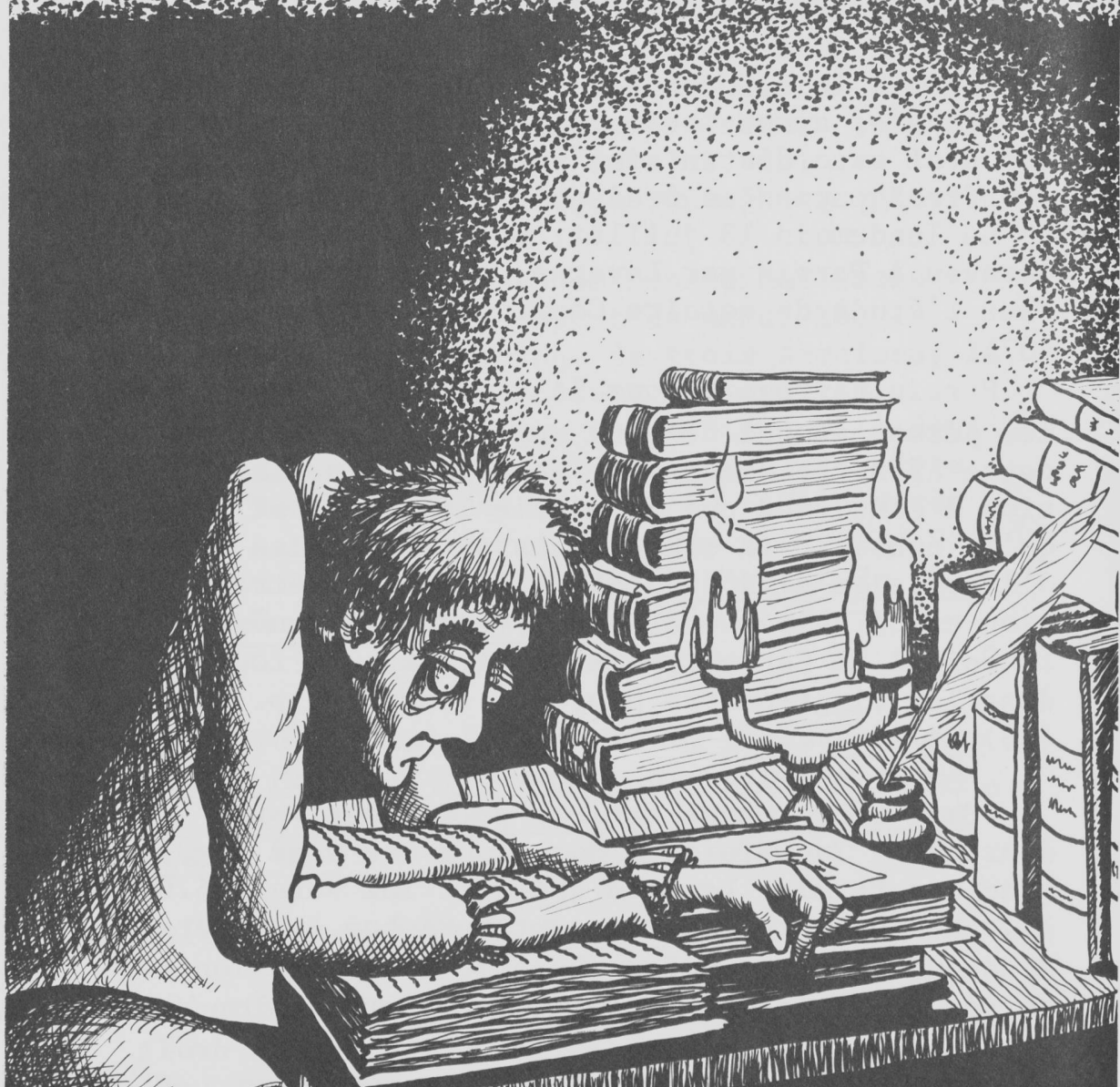

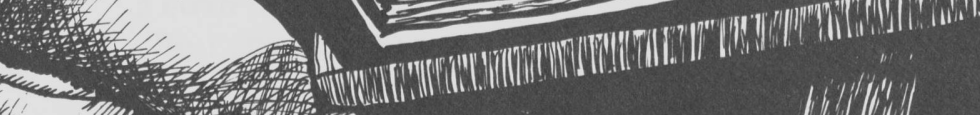

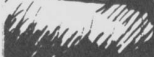

III

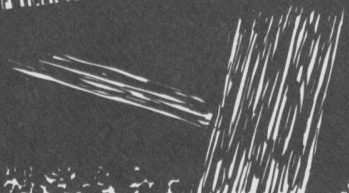

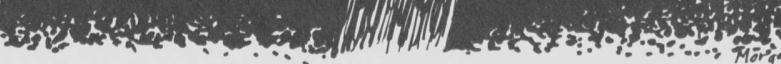

